\title{
A novel grouped sparse representation for face recognition
}

\author{
Jingcheng $\mathrm{Ke}^{1,2,3} \cdot$ Yali Peng ${ }^{1,2} \cdot$ Shigang Liu ${ }^{1,2,3}$ • \\ Zengguo Sun ${ }^{1,2,3} \cdot$ Xili Wang ${ }^{1,2,3}$
}

Received: 24 November 2017 / Revised: 7 May 2018 / Accepted: 15 June 2018 /

Published online: 15 August 2018

(C) The Author(s) 2018

\begin{abstract}
Grouped sparse representation classification methods (GSRCMs) have been attracted much attention by scholars, especially in face recognition. However, pervious literatures of GSRCMs only fuse the scores from different groups to classification the test sample, not consider relationships of the groups. Moreover, in real-world application, many methods of face recognition cannot obtain satisfied recognition accuracies because of the variation of poses, illuminations and facial representations of face image. In order to overcome above-mentioned bottlenecks, in this paper, we proposed a novel grouped fusion-based method in face recognition. The proposed method uses the axis-symmetrical property of face to designs a framework and perform it on original training set to generate a kind of virtual samples. The virtual samples are able to reflect the possible change of face images. Meanwhile, to consider the relationship of different groups and strengthen the representation capability of test sample, the proposed method exploits a novel weighted fusion approach to classify the test sample. Experimental results on five face databases demonstrate that our
\end{abstract}

Yali Peng

431028981@qq.com

Jingcheng Ke

freedom6927@163.com

Shigang Liu

shgliu@snnu.edu.cn

Zengguo Sun

sunzg@snnu.edu.cn

Xili Wang

wangxili@snnu.edu.cn

1 Key Laboratory of Modern Teaching Technology, Ministry of Education, Xi'an 710062, China

2 Engineering Laboratory of Teaching Information Technology of Shaanxi Province, Xi'an 710119 Shaanxi, China

3 School of Computer Science, Shaanxi Normal University, Xi'an 710119, China 
method is reasonable and can obtain higher recognition rate than the other 11 state-of-the-art methods.

Keywords Grouped sparse representation classifications · Axis-symmetrical property · Framework · Virtual sample $\cdot$ Weighted fusion approach

\section{Introduction}

In all biometric techniques, face recognition is the most attractive technique. A lot of face recognition methods have been applied to identity authentication and security system $[3,4,11,21,23]$. However, face recognition still faces a lot of challenges such as the various lightings, facial expressions, poses and environments [10, 13, 14, $33,35,42,54,57,65,68]$. In order to overcome these challenges, a lot of representation-based classification methods (RBCMs) [15, 29, 31, 37, 52, 53, 63, 64, 68] are proposed such as SRC [52], collaborative representation classification (CRC) [63], two-phase test sample representation (TPTSR) [53], linear regression classification (LRC) [37], feature space representation method [61], an improvement to the nearest neighbor (INNC) classification [55], etc. SRC tries to represent the test sample by an optimal linear combination of the training samples. Then, the test sample is assigned to the class which has the minimum deviation. Since SRC uses the $l_{1}$-regularization to obtain the coefficient vector, SRC is viewed as a $l_{1}$ norm-based representation method. Scholars also proposed $l_{2}$ norm-based representation methods such as CRC, LRC and TPTSR. The same as SRC, CRC uses the combination of all training samples to represent the test sample. But it computes the coefficient vector by $l_{2}$-regularization. The recently proposed LRC is closely related with nearest intra-class space (NICS) method [32]. The major difference between LRC and CRC is that LRC tries to use an optimal linear combination of the training samples of each class to represent the test sample. In other words, it establishes a linear system for each class. TPTSR first eliminates the classes which are far from the test sample. Then, the training samples of the remainder classes are combined to represent the test sample.

Besides the above-mentioned RBCMs, another way to improve the recognition rate is simultaneously using the original training sample and corresponding virtual samples $[16,34,38,40,44,46,47]$ to recognize the test sample. In real-world application of face recognition, because of the limited training samples, face recognition methods often suffer the challenges of varying poses, illuminations and facial expressions of face image. To solve these problems, a lot of methods have been proposed. For example, $\mathrm{Xu}$ et al. use the symmetry of the face to generate the mirror samples [57]. Then, the mirror samples are integrated into original training set to recognize the test samples. Another method [58] of $\mathrm{Xu}$ et al. generates a kind of virtual samples by the combination of multiple descriptions or representations. Then, the original training samples and virtual samples are integrated to recognize the test sample. The formula of multiple representations is defined as $\mathbf{J}_{i j}=\mathbf{I}_{i j} \cdot\left(m-\mathbf{I}_{i j}\right)$. In this case, $m=255, \mathbf{I}_{i j}$ and $\mathbf{J}_{i j}$ stand for the intensity of the pixel at the $i-t h$ row and $j-t h$ col. of original training sample and virtual training sample matrix. Experimental results in $[57,58]$ demonstrate that the virtual samples are able to reflect the possible changes of poses, illuminations and facial expressions of face image. Although virtual samples are able 
to enlarge original training set, this might adversely affect the efficiency of the appointed algorithm. So how to improve the recognition rate with limited training samples is still a hot research topic.

To improve the recognition rate with limited training samples, scholars proposed the fusion theory. Fusion theory is mainly performed at three levels, i.e., score level, decision level and feature level. Although the decision level fusion is very easy to implement, the multisource information cannot be fully exploited. Feature level fusion is able to exploit the most information from the original data, but it need to overcome the challenges of the inconsistence of different data set. For the score level fusion [8, 20, 68], there are three kinds of score level fusions, i.e., transformation-based score fusion [30], classifier-based score fusion [26] and density-based score fusion. The classifier-based score fusion directly combines the scores of different data source into form a feature vector. Then, the test sample is classified in teams of the feature vector. The transformation-based score fusion first transforms the scores of different data source into a common domain. Then, it classifies the test sample by the integration of the normalized scores. Densitybased score fusion is able to obtain higher recognition rate than transformation-based score fusion and classifier-based score fusion if we can accurately evaluate the scores densities. But in real-world applications, because of the complex of evaluation, densitybased score fusion is hard to use. Pervious proposed literatures [11, 12, 18, 49] reflect that if the score fusion methods were integrated into RBCMs, especially the GSRCMs $[48,56,62]$, the test sample is more possibly to be assigned to the correct class.

However, pervious proposed GSRMs only consider the residuals from different kinds of groups, and not consider the relationship between group and group. Moreover, in practical application of face recognition, we only have very small number of training samples to use. So this motivates us to propose a novel GSRM to resolve above problems. In order to classify the test sample, the proposed GSRM first selects the training samples of its nearest classes to form the first group. Next, a framework is performed on the first group to generate a kind of virtual samples. All virtual samples are formed the second group. Then, SRC is performed on the two groups to generate two residuals for each class. Finally, two residuals of a class and their distance are fused as the ultimate residuals. The distance is defined as the difference between the reconstructed sample of the class from the first group and second group.

The proposed method has the following main contributions. Firstly, it exploits the idea that representing the test sample by its nearest neighbor classes. This is able to reduce the side-effect of some low relative training samples. Secondly, by designing a framework of generating a kind of virtual sample, the challenges of the various poses, illuminates and facial expressions can be partly overcame. Thirdly, for the classification decision of the test sample, the proposed method not only takes into count two residuals of a class, but also considers a distance of the class since they all contain discriminant information. Fourthly, a novel weighted fusion approach is proposed to fuse two residuals and the distance of the class. The fusion approach takes the test sample into account and generates adaptive weights to different residuals. This is helpful to better classify the test sample.

In the rest of this paper, we mainly give a brief review to SRC and LRC in section 2. In section 3, we present the details of the proposed method. In the meanwhile, the rationalities of the proposed method are analyzed in section 4. In section 5, we conduct the experiments on five databases. The conclusion is showed in section 6 . 


\section{Related works}

SRC and LRC are two conventional representation-based methods. Many proposed representation-based classification methods are based on SRC and LRC. Assuming that there are $c$ classes and each class has $n$ training samples. The test sample is denoted by $z$. Moreover, we converted each sample matrix into a column vector.

\subsection{SRC}

Suppose that the samples of the $i$-th class are denoted by $x_{i 1}, x_{i 2}, \ldots, x_{i n}$, where $i=1,2, \ldots, c$. Let us combine all training samples to form a matrix $\mathbf{X}=\left[x_{11}, \ldots, x_{1 n}, \ldots, x_{c 1}, \ldots x_{c n}\right]$. According to SRC, we have

$$
\mathbf{X} A \approx z
$$

where $A=\left[a_{11}, \ldots, a_{1 n}, \ldots, a_{c 1}, \ldots a_{c n}\right]$ is the coefficient vector. Next, SRC solves the Eq. (1) with $l_{1}$-norm regularization

$$
A^{\prime}=\operatorname{argmin}\|A\|_{1}, \text { s.t. }\|z-\mathbf{X} A\|_{2}^{2} \leq \xi
$$

In this case, $\xi$ is very small positive.

Assuming that the $a_{e}^{\prime}, \ldots, a_{f}^{\prime}$ is the coefficients of the $i$-th class, then the representation residual of the $i$-th class can be written as

$$
r_{i}=\left\|z-\sum_{t=e}^{f} a_{t}^{\prime} x_{i t}\right\|
$$

If the minimum residual is from the $i$-th class, the test sample will be classified to the $i$-th class.

The major difference between CRC and SRC is that CRC solves the Eq. (2) with $l_{2}$-norm regularization.

\subsection{LRC}

LRC is viewed as a $l_{2}$ norm-based representation method. Let us combine all training samples of the $i$-th class to form a matrix $\mathbf{X}_{i}=\left[x_{i 1}, \ldots, x_{i n}\right]$. LRC represents the test sample by the linear combination of the training samples of each class. In other words, it exploits the following model to obtain the representation coefficient of each training sample.

$$
\mathbf{X}_{i} B_{i} \approx z
$$

where $B_{i}=\left[b_{i 1}, b_{i 2}, \ldots, b_{i n}\right]$ is the coefficient vector of the $i$-th class. The model of solving Eq. (4) can be written as

$$
B_{i}^{\prime}=\left[b_{i 1}^{\prime}, \ldots, b_{i n}^{\prime}\right]=\left(\mathbf{X}_{i}^{T} \mathbf{X}_{i}+\mu \mathbf{I}\right)^{-1} \mathbf{X}_{i}^{T} z
$$


In this case, the residual of the $i$-th class is

$$
d_{i}=\left\|z-\sum_{t=1}^{n} b_{t}^{\prime} x_{i t}\right\|
$$

The test sample will be assigned to the $i$-th class if the minimum residual is from the $i$-th class.

\section{The proposed method}

\subsection{The description of framework}

In this subsection, we show the details of the framework. Let us denote the left and right vector of an original face image as $z_{1}$ and $z_{2}$. The average vector of $z_{1}$ and $z_{2}$ is denoted by $z_{0}$. Both $z_{1}$, $z_{2}$ and $z_{0}$ are column vectors. The model of obtaining $z_{1}, z_{2}$ and $z_{0}$ is as follows. Let $\mathbf{F}_{j}(j=1,2$, ..., $Q$ ) denotes the $j$-th column of the 2D image matrix, where $Q$ is an even number. The pixel at the $i$-th row and $j$-th column of $\mathbf{F}$ is denoted by $\mathbf{F}_{i j} . z_{1}, z_{2}$ and $z_{0}$ are defined as

$$
z_{1}=\left[\begin{array}{c}
\mathbf{F}_{1} \\
\mathbf{F}_{2} \\
\vdots \\
\mathbf{F}_{\frac{Q}{2}}
\end{array}\right], z_{2}=\left[\begin{array}{c}
\mathbf{F}_{Q} \\
\mathbf{F}_{Q-1} \\
\vdots \\
\mathbf{F}_{\frac{Q}{2}+1}
\end{array}\right], z_{0}=\frac{z_{1}+z_{2}}{2}
$$

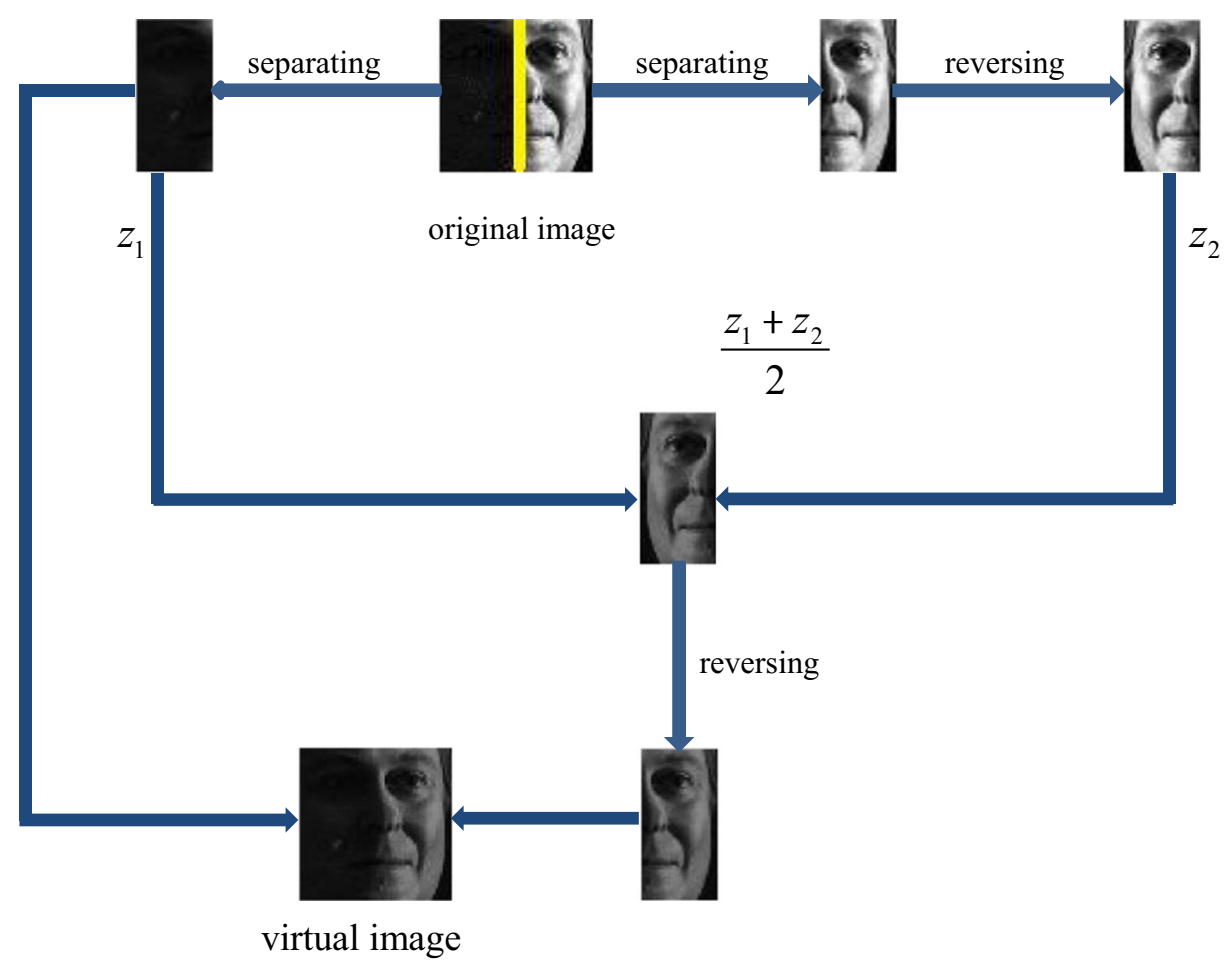

Fig. 1 The flow chart of the procedure 
Finally, $z_{1}$ and $z_{0}$ are integrated to form a kind of virtual image. The procedure of the framework is showed in Fig. 1.

\subsection{The description of novel weighted fusion approach}

In this subsection, we show the details of the novel weighted fusion approach. It is sure that conventional weighted fusion approaches are able to take advantages of data from different source. However, conventional weighted fusion approaches assign fix weights to different data. Sometimes the fix weights are not the optimal weights of the data. So this motives us to propose an automatic and adaptive weighted fusion approach. Our weighted fusion approach supposes that there are two training sample sets and each set has $c$ classes. The residual between a test sample and the $i$-th class of the first set is denoted by $r_{i}^{1}, i=1,2, \ldots, c$. The residual between the test sample and the $i$-th class of the second set is denoted by $r_{i}^{2}$. Moreover, we define $\alpha_{h}=\frac{\max \left(r_{1}^{h}, r_{2}^{h}, \ldots, r_{c}^{h}\right)-\min \left(r_{1}^{h}, r_{2}^{h}, \ldots, r_{c}^{h}\right)}{q}$, where $q=\sum_{t=1}^{c} r_{t}^{h}$ and $h=1,2$. The details are as follows.

Step 1: $\quad r_{i}^{1}$ and $r_{i}^{2}$ are normalized to the range of 0 to 1 . In this case, $r_{i}^{1}$ and $r_{i}^{2}$ can be rewritten as

$$
\begin{aligned}
& r_{i}^{1}=\left(r_{\text {max }}^{1}-r_{i}^{1}\right) /\left(r_{\text {max }}^{1}-r_{\text {min }}^{1}\right) \\
& r_{i}^{2}=\left(r_{\text {max }}^{2}-r_{i}^{2}\right) /\left(r_{\text {max }}^{2}-r_{\text {min }}^{2}\right)
\end{aligned}
$$

Step 2: $\quad r_{1}^{1}, r_{2}^{1}, \ldots, r_{c}^{1}$ are sorted in the order of ascending and the sorted results is recorded as $r_{1}^{1^{\prime}} \leq r_{2}^{1^{\prime}} \leq \cdots \leq r_{c}^{1^{\prime}}$. After that, $r_{1}^{2}, r_{2}^{2}, \ldots, r_{c}^{2}$ are sorted in the order of ascending and the sorted result is recorded as $r_{1}^{2^{\prime}} \leq r_{2}^{2^{\prime}} \leq \cdots \leq r_{c}^{2^{\prime}}$. Let $w=\left(r_{2}^{1^{\prime}}-r_{1}^{1^{\prime}}\right)+\left(r_{2}^{2^{\prime}}-r_{1}^{2^{\prime}}\right)$, $w_{1}=\frac{\left(r_{2}^{1}-r_{1}^{\prime}\right)}{w}, w_{2}=\frac{r_{2}^{2^{\prime}}-r_{1}^{2^{\prime}}}{w}$. So, the ultimate residual of the $i$-th class can be written as

$$
f_{i}=\alpha_{1} w_{1} r_{i}^{1}+\alpha_{2} w_{2} r_{i}^{2}
$$

\subsection{The description of the grouped spare classification}

The details of the proposed method are showed in this subsection. The number of the classes is still defined as $c$. Each class has $n$ training samples. All sample matrixes are converted into column vectors. Each column vector has $m$ components. The training samples of the $i$-th class are denoted by $x_{i 1}, x_{i 2}, \ldots, x_{i n}$, where $i=1,2, \ldots, c$. And the test sample is denoted by $z$. The details are as follows. 
First step: We first use the linear combination of the training samples of original training set to represent the test sample. According to SRC, each class of the training set obtains a residual. Next, all training samples from $K$ classes with the first $K$ smallest residuals are selected to form the first group, where $K<c$.

Second step: We perform the framework on the first group to generate the virtual samples. The virtual samples are integrated into the second group. Let us respectively combine all training samples from the first and second groups to form the matrixes $\mathbf{G}_{1}$ and $\mathbf{G}_{2}$. Then SRC are performed on these two groups. In this case, we have

$$
\mathbf{G}_{1} A_{1} \approx z, \mathbf{G}_{2} A_{2} \approx z
$$

where $A_{1}$ and $A_{2}$ are the coefficient vectors of these two groups. To solve Eq. (11), according to Eqs (2) and (3), each class of the $K$ selected classes obtains two residuals. And corresponding training samples obtain two coefficients.

Third step: Let $a_{e}, \ldots, a_{f}$ and $a_{e}^{\prime}, \ldots, a_{f}^{\prime}$ be the coefficients of the training samples. Next, the reconstructed samples of the $i$-th class in two groups are defined as $\sum_{t=e}^{f} a_{t} x_{i t}$ and $\sum_{t=e}^{f} a_{t}^{\prime} x_{i t}^{v}$, where $x_{i t}^{v}$ is the virtual sample of $x_{i t}$. Then, the distance of the $i$-th class can be written as

$$
d_{i}=\left\|\sum_{t=e}^{f} a_{t} x_{i t}-\sum_{t=e}^{f} a_{t}^{\prime} x_{i t}^{v}\right\|
$$

Fourth step: Let us perform the proposed weighted fusion approach on two groups. According to the description of subsection 3.2, the ultimate residual of the $i$-th class of $K$ selected classes can be written as

$$
s_{i}=\alpha_{1} w_{1} r_{i}^{1}+\alpha_{2} w_{2} r_{i}^{2}+\left(\alpha_{1}-\alpha_{2}\right) d_{i}
$$

where $r_{i}^{1}$ and $r_{i}^{2}$ are two residuals of the $i$-th class. If $s_{i}$ is the minimum residual, the test sample will be classified to the $i$-th class.

\section{Analysis of the proposed method}

\subsection{Intuitive rationalities of the proposed method}

There are four rationalities in the proposed method. Firstly, the test sample is represented by linear combination of the training samples of its nearest neighbor classes. In representationbased classification method, different training samples have different effect on the classification decision of the test sample. If a training sample is close to the test sample, the training 
sample has a great effect on the decision of classifying the test sample, and if a training sample is far enough from the test sample, the training sample has little effect and even has side-effect on the decision of classifying the test sample. So, in order to better represent the test sample, we can eliminate the training samples of the classes that are far from the test samples. In fact, if the test sample is represented by the linear combination of the training samples of all class, according to SRC, the training samples of its nearest neighbor classes will obtain small representation coefficients. This is not good enhancing the capability of representation. However, if we remove the training samples of the class that are far from the test sample, the representation coefficients of its nearest neighbor classes will be enlarged. In this case, the test sample can be better represented. Secondly, we proposed a framework to generate a kind of virtual sample. In real-world applications, we often suffer the challenges of various poses, illuminations and facial expressions of face image because of the limited training samples. So, the simultaneous use of the original training samples and virtual samples is able to represent the test sample better. For the framework, the obtained virtual samples are not far from original training samples. Moreover, the virtual samples and original training samples are not linear correlative. In other words, the virtual samples are not from the subspace of the original training set. So the generated virtual samples are very helpful to strengthen the capability of representing the test sample. Thirdly, a novel grouped classification method is proposed to classify the test sample. We not only take into count two residuals of a class in the first and second group, but also consider a distance of the class. Two residuals of a class contain the discriminant information of the test sample. For the distance, let us denote $\Delta z_{i}^{1}=z^{-} \sum_{t=e}^{f} a_{t} x_{i t}$ be the residual vector of the $i$-th class from the first group, $\Delta z_{i}^{2}=z^{-} \sum_{t=e}^{f} a_{t}^{\prime} x_{i t}^{v}$ be the residual vector of the $i$-th class from the second group. Figure 2 shows the relationship of $\Delta z_{i}^{1}, \Delta z_{i}^{2}$ and $d i s_{i}=\sum_{t=e}^{f} a_{t} x_{i t}-\sum_{t=e}^{f} a_{t}^{\prime} x_{i t}^{v}$. Clearly,

$$
d i s_{i}=\Delta z_{i}^{1}-\Delta z_{i}^{2}=\sum_{t=e}^{f} a_{t} x_{i t}-\sum_{t=e}^{f} a_{t}^{\prime} x_{i t}^{v}
$$

It reflects the difference between the reconstructed samples of the class in the first and second group. In addition, $\Delta z_{i}^{1}, \Delta z_{i}^{2}$ and $d i s_{i}$ form a triangle of the $m$-dimensional space, i.e, subsection 3.3. $\Delta z_{i}^{1}$ and $\Delta z_{i}^{2}$ show the discriminant information between the test sample and $i$ th class, so the distance $d i s_{i}$ simultaneously contains the discriminant information of the test sample in two groups. If the reconstructed sample of the class in the first group is similar to the one in the second group, the corresponding distance will be very small. In this case, the fusion

Fig. 2 The relationship of $\Delta z_{i}^{1}, \Delta$ $z_{i}^{2}$ and dis $_{i}$

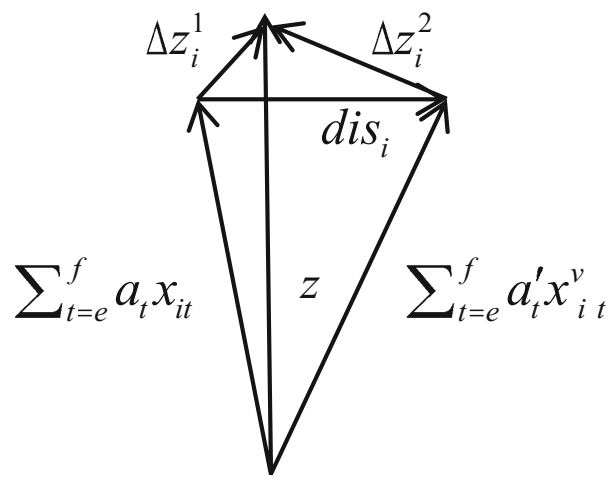


two kinds of residuals and the distance of the class maybe obtain smaller residual. This helps improve the recognition accuracy of test sample. Fourthly, a novel weighted fusion approach is proposed to fuse two residuals and the distance of a class. The weighted fusion approach uses a simple way to generate the weights for different residuals of a class automatically. In other words, the weighted fusion approach takes each test sample into account and determines optimal weights for the test sample. This is able to consider the dissimilarity between the test sample and the class flexibly.

\subsection{More analysis of the proposed method}

\subsubsection{Insight into the advantage of the framework of our method}

In this subsection, we compare our method with the method [54] to demonstrate that the linear combination of virtual samples of our method is able to better represent the test sample. The method [54] exploits the symmetry of face to generate a kind of axis-symmetrical virtual sample. It first divides each training face image into two halves. Next, the two halves are used to generate two symmetrical virtual face images. All original training samples and virtual samples are combined to form the first and second group, respectively. Then, one of conventional RBCs is performed on these two groups to generate two residuals for each class. Finally, two residuals of a class are fused to classify the test sample. The details on how to obtain the virtual sample of the method [54] are showed in Fig. 3. For the comparison, we convert all sample into column vectors and use Euclidean distance $d=\sqrt{(x-y)}{ }^{T}(x-y)$ to evaluate the similarly between the test sample and reconstructed virtual sample of the method [54], reconstructed virtual sample of our method. The reconstructed virtual sample is defined as the linear combination of virtual samples from assigned class. In Fig. 4, the first row is the test samples of YALEB face database [17] from the 1-th, 2-th, 3-th, 4-th and 5-th class. The second and third rows are the reconstructed virtual samples of the method [54] and our method from the first five classes. According to the evaluation results in Table 1, we conclude that the distances between the test samples and reconstructed virtual samples of our method are smaller than the distances between the test samples and reconstructed virtual samples of the method [54]. In other words, the reconstructed virtual samples of our method are closer to the test samples. This implies that the integration of original training samples and virtual samples of our method is able to better recognize the test sample.

\subsubsection{The advantage of the weighted fusion approach of our method}

It should be note that RBCMs classify the test sample based on the residual of each class and assign the test sample to the class which has the smallest residual. The residual reflects the representation capability of each class to the test sample. In the four step of our method, we compare the proposed weighted fusion approach with fix weighted fusion approach to demonstrate that the proposed weighted fusion approach is able to better classify the test sample. We first give a toy example to demonstrate the advantage of the proposed weighted fusion approach. Let us select the test sample from the 5-th class in YALEB face database. The parameter $K$ of our method is set to 19 . Moreover, the number of the training samples of each class is set to 4 . For the fix weighted fusion approach, the coefficients from the fourth step of our method are set to $0.4,0.4$ and 0.2. In Fig. 5, we conclude that the proposed weighted fusion 


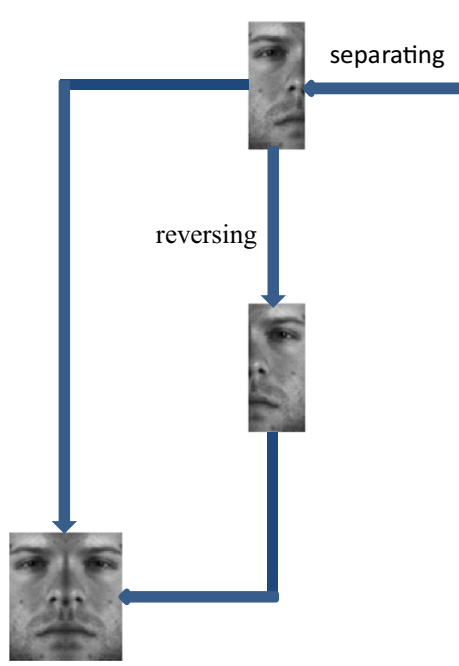

virtual sample with

left halve

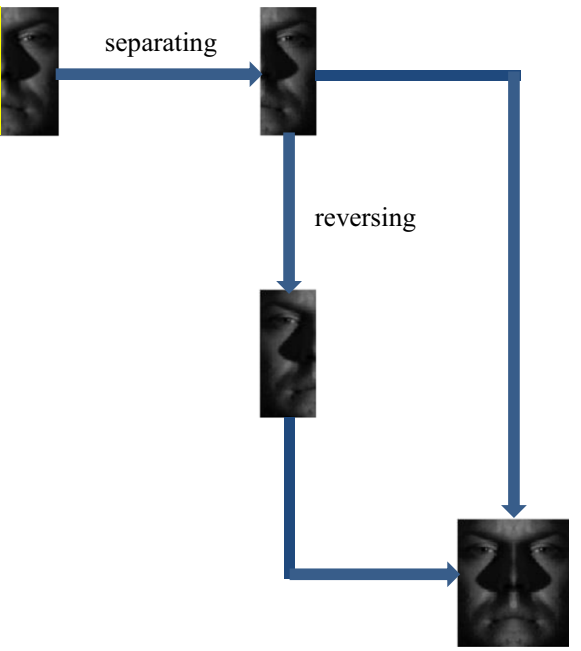

virtual sample with

right halve

Fig. 3 The details of the method [54]

approach is able to obtain smaller residuals than the fix weighted approach. Clearly, the discriminability of red line is better than blue line. In red line, it is easy to determine that the smallest residual is from the 5-th class. But in blue line, the residuals of the 1-th class and 4-th class are approximately on the same horizontal line. Moreover, using the fix weighted fusion approach cannot classify the test sample to correct class. It classifies the test sample to the 4-th class, not the 5-th class. However, our method is able to classify the test sample to the correct class because the smallest residual is from the 5-th class. In this case, compared with fix weighted fusion approach, our weighted fusion approach is able to better classify the test sample.
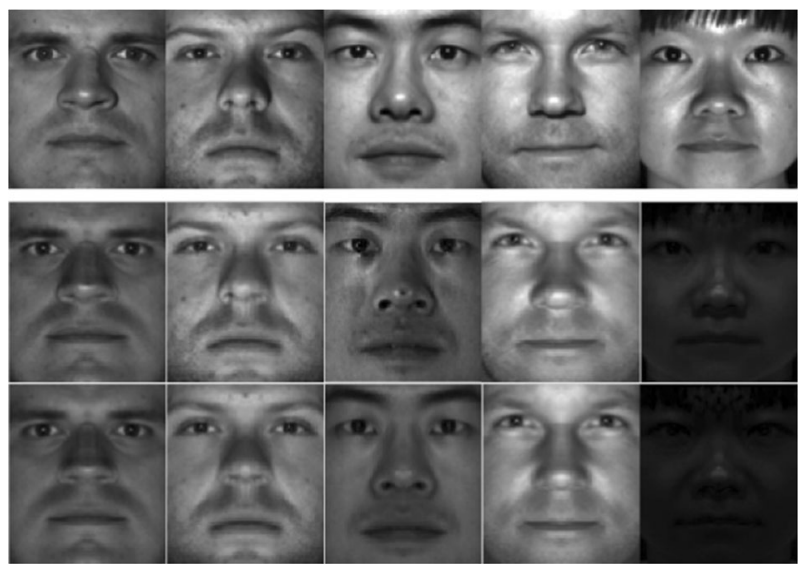

Fig. 4 The first, second and the third rows are the test samples, reconstructed virtual samples of method [54] and reconstructed virtual samples of our method. 
Table 1 The Euclidean distances between the test sample and reconstructed virtual sample of our method, reconstructed virtual sample of method [54]

\begin{tabular}{llllll}
\hline No. of the subject & 1 & 2 & 3 & 4 & 5 \\
\hline Reconstructed virtual samples of the method [54] & 0.2172 & 0.6450 & 0.2707 & 0.28540 .8696 \\
Reconstructed virtual samples of our method & 0.1763 & 0.4495 & 0.2390 & 0.27180 .6520 \\
\hline
\end{tabular}

We further use CMU-PIE face database as an example to demonstrate the advantage of the proposed weighted fusion approach. The test sample is selected from the 2-th class and the parameter $K$ of our method is set to 19 . Moreover, we took the first 4 samples of each class as training samples. For the fix weighted fusion approach, the coefficients of the fourth step of our method are set to $0.4,0.4$ and 0.2. In Fig. 6, it is obvious that the residuals of the proposed weighted approach are smaller than the residuals of the fix weighted approach. The residual of the 2-th class is clearly the smallest in red line. Meanwhile, the use of fix weighted approach erroneously classifies the test sample to the 6-th class because the 6-th class has the smallest residual value. However, our method is able to classify the test sample to the 2-th class. This further implies that the representation ability of our method is better than the use of fix weighted approach.

\subsubsection{The comparison between our weighted fusion approach and the method [67]}

The method [67] proposed an adaptive weighted fusion approach (ADWF) for face recognition. ADWF takes each test sample into account and uses two variables to generate the weights for a class automatically. Suppose that each class has $u$ residuals, the first variable is defined as

$$
\delta_{k}=\frac{\operatorname{sumd}-\min \left(r_{1}^{k}, r_{2}^{k}, \ldots, r_{c}^{k}\right)}{\text { sumd }}
$$

where sumd $=\sum_{t=1}^{u} r_{i}^{t}$ and $k=1,2,3, \ldots, u$. For the second variable, ADWF firsts sorts the residuals $r_{i}^{1}, r_{i}^{2}, \ldots, r_{i}^{u}$ in the order of ascending and the results are described as $r_{1}^{\prime 1} \leq r_{2}^{\prime} \leq \cdots \leq r_{c}^{\prime 1}, r_{1}^{\prime 2} \leq r_{2}^{\prime 2} \leq \cdots \leq r_{c}^{\prime 2}, \ldots, r_{1}^{\prime u} \leq r_{2}^{\prime u} \leq \cdots \leq r_{c}^{\prime u}$. Then, the second variable is defined as

$$
w_{k}=\frac{r_{2}^{\prime k}}{w}
$$
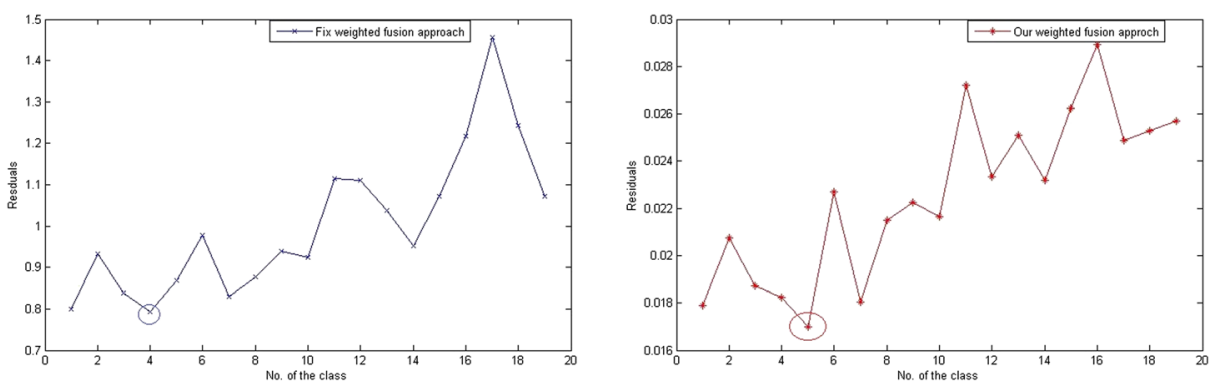

Fig. 5 The residuals of the fix weighted fusion approach and our weighted fusion approach in YALEB face database 

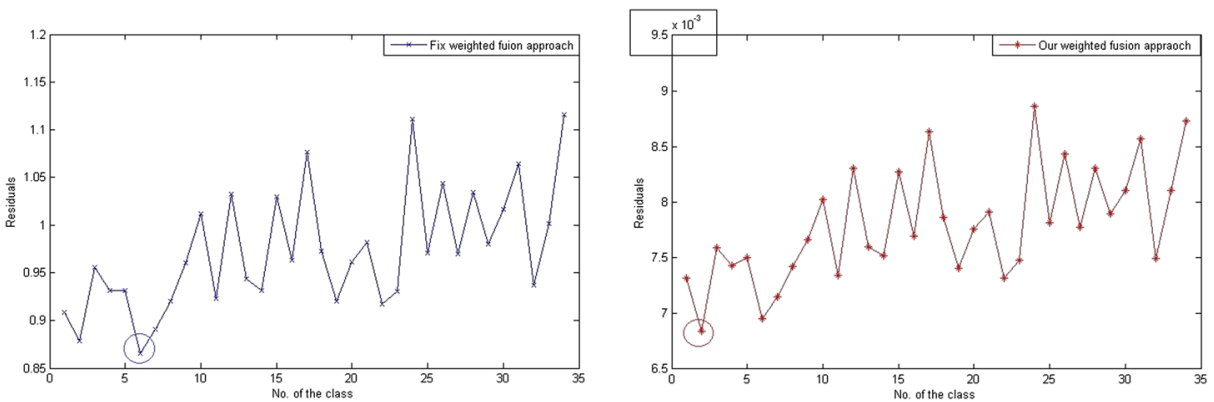

Fig. 6 The residuals of the fix weighted approach and our method in CMU-PIE face database

where $w=\sum_{t=1}^{u} r_{2}^{\prime t}$. Finally, the residual of the $i$-th class is denoted as

$$
s_{i}^{\prime}=\delta_{1} w_{1} r_{i}^{1}+\delta_{2} w_{2} r_{i}^{2}+\cdots+\delta_{u} w_{u} r_{i}^{u}
$$

The same as above-mentioned comparison strategy, we compare our weighted fusion approach and ADWF in the four step of our method. In this subsection, we select the test sample from the 1-th class in CMU-PIE face database. In the meanwhile, we set the parameter $K$ of our method and the number of the training samples of each class to 0.01 and 27. In Fig. 7 , red line shows the residuals of our weighted fusion approach and blue line shows the residuals of ADWF. It is obvious that the residuals of our weighted fusion approach are smaller than ADWF. Meanwhile, in blue line, it is hard to discriminate that which class has the smallest the residual. However, obviously, the residual of the 1-th class is smallest in red line. Moreover, ADWF cannot classify the test sample to the correct class. The smallest residual is from the 3th class. However, our method is able to classify the test sample to the correct class. The smallest residual of our weighted fusion approach is from the first class. So the results show that the discriminability and representation capability of the test sample of our weighted fusion approach are stronger than ADWF.

To further demonstrate that our weighted fusion approach enable better representing the test sample than ADWF, we use FERET face database an example. In this case, the test sample is from the 38-th class. In the meanwhile, we set the parameter $K$ and the numbers of the training samples set to 80 and 3. The comparison result is showed in Fig. 8. Clearly in Fig. 8, the residuals of our weighted fusion approach are smaller than ADWF. In red line, it is obvious that the residual of the 38-th class is the smallest. But hard to discriminate that which class has the smallest residual in blue line. Moreover, our weighted fusion approach is able to classify
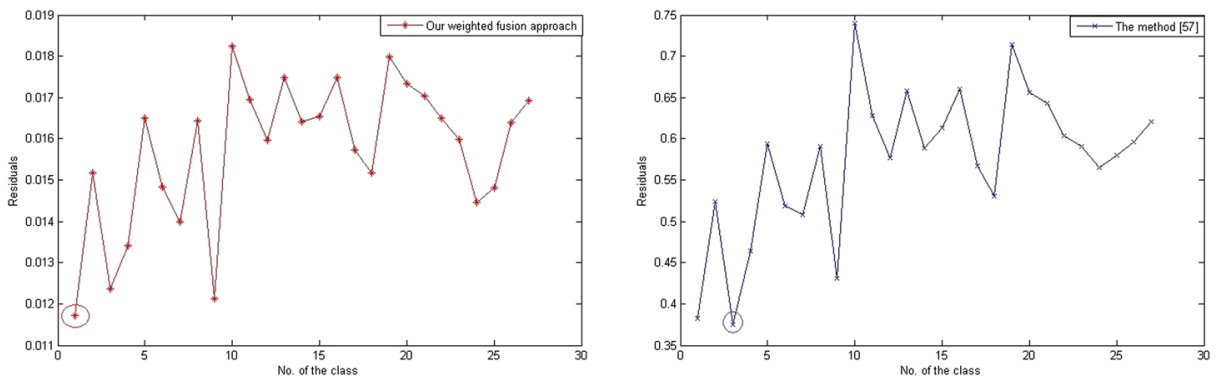

Fig. 7 The residuals of our weighted fusion approach and ADWF in CMU-PIE face database 

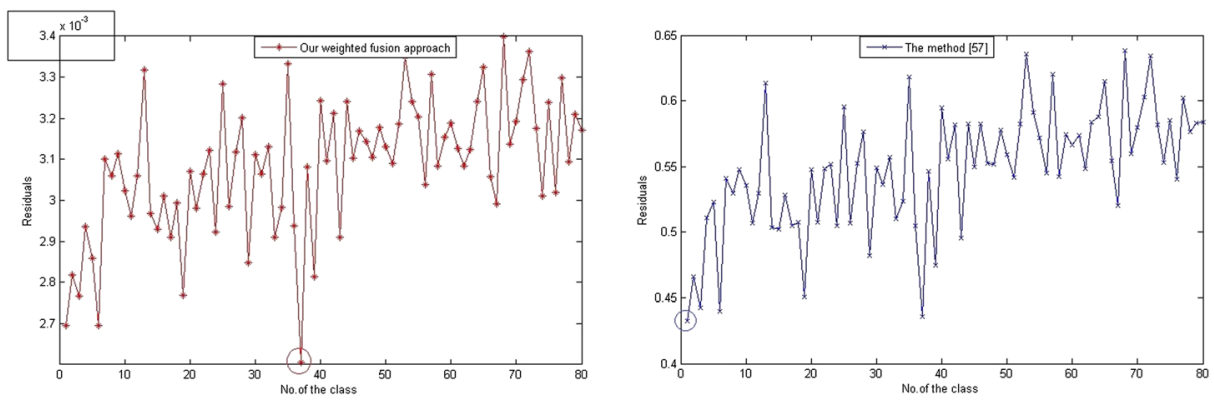

Fig. 8 The residuals of our weighted fusion approach and ADWF in FERET face database

the test sample to the correct class. The smallest residual of our weighted fusion approach is from the 38-th class. However, the smallest residual of ADWF is from the 1-th class, not from the 38-th class. This further shows that our weighted fusion approach is able to better represent the test sample than ADWF.

\subsubsection{Insight into the advantage of the distance of our method}

In this subsection, we use an example to demonstrate that the distance of a class helps improve the recognition accuracy of test samples. We first selected the test sample from the 8-th class in ORL face database. The parameter $K$ and the number of the training sample of each class are set to 20 and 4. Red and blue lines in Fig. 9 reflect the ultimate residuals generated by the fusion of two residuals and by our method. For the red line, it is obvious that the ultimate residual of the 8-th class is the smallest. However, it is hard to discriminate which class has the smallest residual in blue line. The residuals of the 25-th class and the 8-th class are approximately at the same level. Moreover, the simple fusion of two residuals cannot classify the test sample to the correct class. The smallest fusion residual is from the 25-th class rather than the 8 -th class. This implies that our method is more discriminative than the simple fusion of two residuals.

Next, we use Libor face database as a sample to further demonstrate that the distance of a class is able to better recognize the test sample. The test sample is selected from the 12-th class. The parameters $K$ and the number of training samples of each class are set to 76 and 4 . Fig. 8 shows the residuals between the test sample and the classes of Libor face database. In Fig. 10,

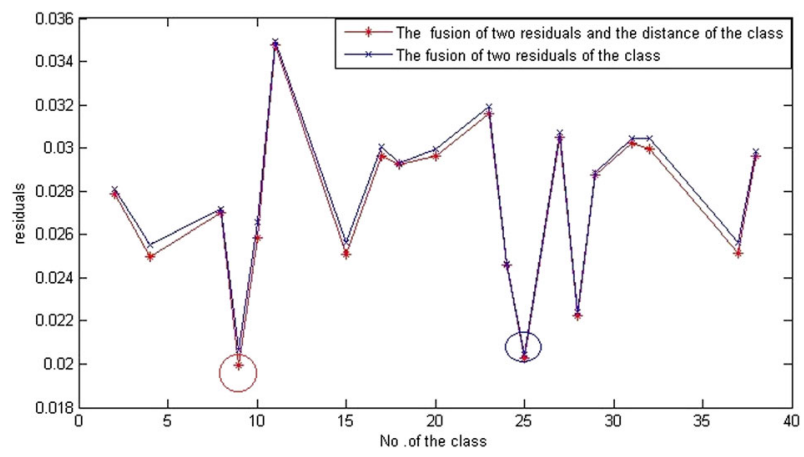

Fig. 9 The fusion results of two residuals and results of two residuals and corresponding distance in ORL face database 


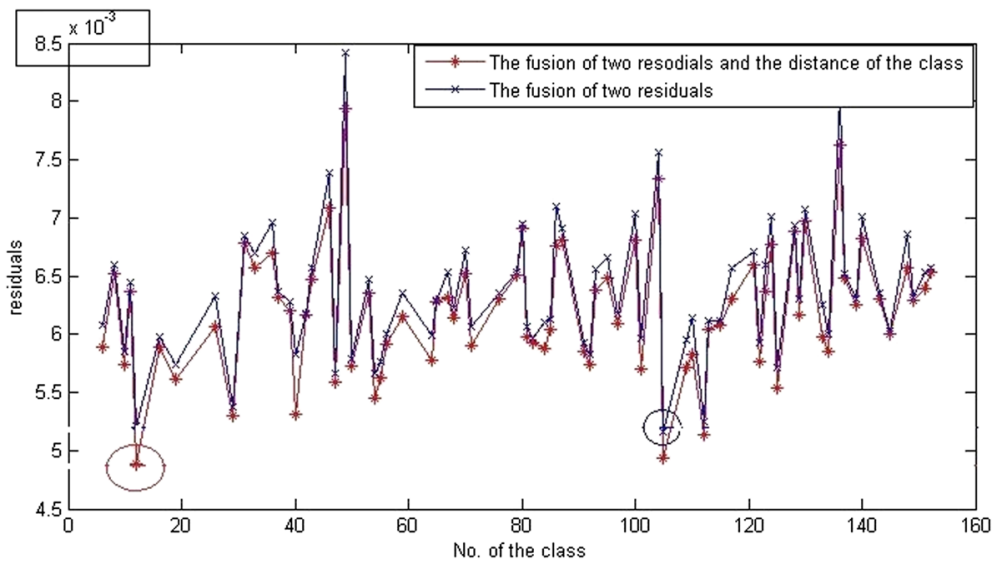

Fig. 10 The fusion results of two residuals and results of two residuals and corresponding distance of the class in Libor face database

it is clearly that the residual of the 12-th class is the smallest in red line. However, the smallest residual in blue line is not obvious. In the meanwhile, the test sample cannot be accurately classified by the simple fusion of two residuals, because the smallest residual of the class is from the 106-th class, not from the 12-th class. This further implies that the discriminability of our method is stronger than the simple fusion of two residuals.

\section{Experimental result and discussion}

In this section, the ORL, FERET, Georgia Tech and PIE face databases were used to conduct the experiment. In the meanwhile, SRC, CRC, LRC, coarse to fine $K$ nearest neighbor classification (CFKNNC) [56], improvement to nearest neighbor classification (INNC), homotopy [7], primal augmented lagrangian method (PLAM) [60], the method [65], discriminative sparse representation method (DSRM) [59], block-diagonal representation (BDLRR) [66] and the method [27] were used to compare with our method. Moreover, we set the parameter $\mu$ of our method as 0.01 . And the number of iteration of Homotopy and PLAM is set to 10 . For the parameter $K$, the size is various because the number of the classes of different datasets is different. In the middle of the experiments, we find that the improvement of recognition accuracy of our method is obvious if $K$ approximately equals to one-half or onethird of the number of the classes. Finally, for the computation formula of accuracy, assuming that the number of the test samples in correct classes is $p_{r}$, then the formula is define as accuracy $=\frac{p_{r}}{C \times n} \times 100 \%$.

\subsection{Experiments on ORL face database}

We first conduct the experiments on a subset of ORL face database [41]. The subset has 400 images from 40 classes and each class has 10 images. The size of each image is $30 \times 30$. Some training and test images of the first three classes are showed in Fig. 11. Moreover, we used the first 1, 2, 3, 4 images of each class as the training images and the remainder samples as the test images. $K$ in our method is set to 20 , respectively. The experimental results were showed in Table 2. We find that our method performs better than SRC, LRC, CRC, CFKNNC, INNC, 

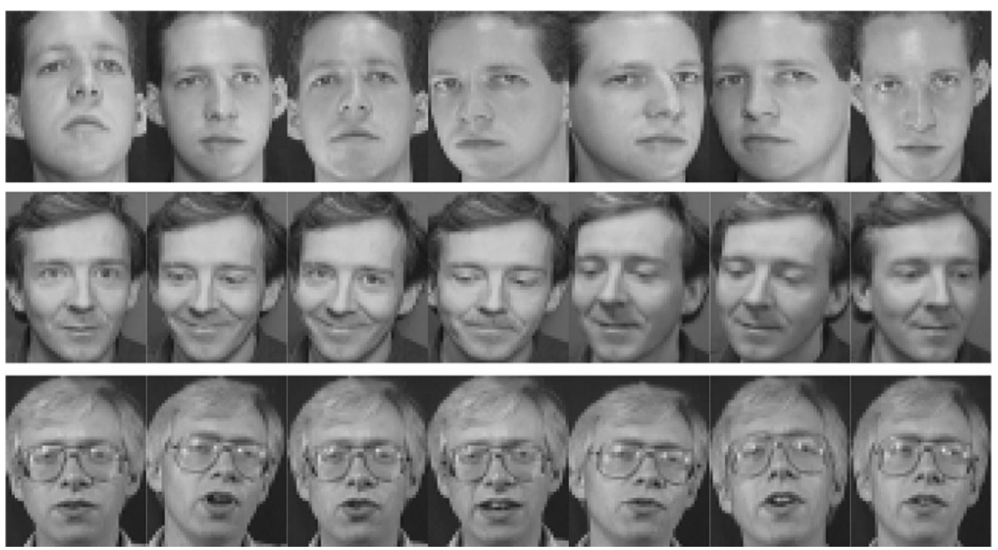

Fig. 11 Some training and test images of the first three classes

homotopy, PLAM, the method [54], DSRM, BDLRR and the method [27]. For example, when the number of training images per class is 4, SRC, LRC, CRC, CFKNNC, INNC, homotopy, PLAM, the method [54], DSRM, BDLRR, the method [27] and the proposed method are $90 \%$, $85 \%, 89.17 \%, 82.52 \%, 81.25 \%, 89.75 \%, 86.25 \%, 77.50 \%, 92.17 \%, 90.42 \%, 84.17$ and $92.50 \%$, respectively. The accuracies of all other methods are lower than that of the proposed method.

\subsection{Experiments on Georgia Tech face database}

In this subsection, we conducted the experiments on the Georgia Tech face database [19]. The Georgia Tech face database has 750 images from 50 faces. Each face has 15 images. The first 1, 2, 3 and 4 images of each face were selected as training images and the rest samples as test images. In the meanwhile, we set the parameter $K$ to 15 . Figure 12 shows some training images and test images of Georgia Tech face database. We respectively used SRC, LRC, CRC, CFKNNC, INNC, homotopy, the method [54], PLAM, the method [27] to compare with our method and obtained the experimental results shown in Table 3. In Table 3, we conclude that the recognition rates of our method are higher than those eleven state-of-the-art methods.

Table 2 THE ACCURACIES (\%) ON THE ORL FACE DATABASE

\begin{tabular}{llllr}
\hline No. of the training sample & 1 & 2 & 3 & 4 \\
\hline SRC & 67.50 & 85.00 & 85.17 & 90.00 \\
LRC & 67.50 & 79.06 & 81.79 & 85.00 \\
CRC & 68.06 & 83.44 & 86.07 & 89.17 \\
INNC & 68.06 & 78.75 & 78.21 & 82.50 \\
CFKNNC & 69.47 & 82.19 & 80.00 & 82.52 \\
Homotopy & 64.17 & 81.88 & 87.53 & 89.75 \\
PLAM & 64.72 & 79.38 & 84.29 & 86.25 \\
The method [54] & 66.11 & 75.62 & 78.57 & 77.50 \\
DSRM & 73.06 & 85.31 & 88.93 & 92.33 \\
BDLRR & 65.56 & 80.13 & 87.86 & 90.42 \\
The method [27] & 65.83 & 81.25 & 83.21 & 84.17 \\
Our method & $\mathbf{7 5 . 0 0}$ & $\mathbf{8 8 . 4 4}$ & $\mathbf{8 9 . 6 4}$ & $\mathbf{9 2 . 5 0}$ \\
\hline
\end{tabular}



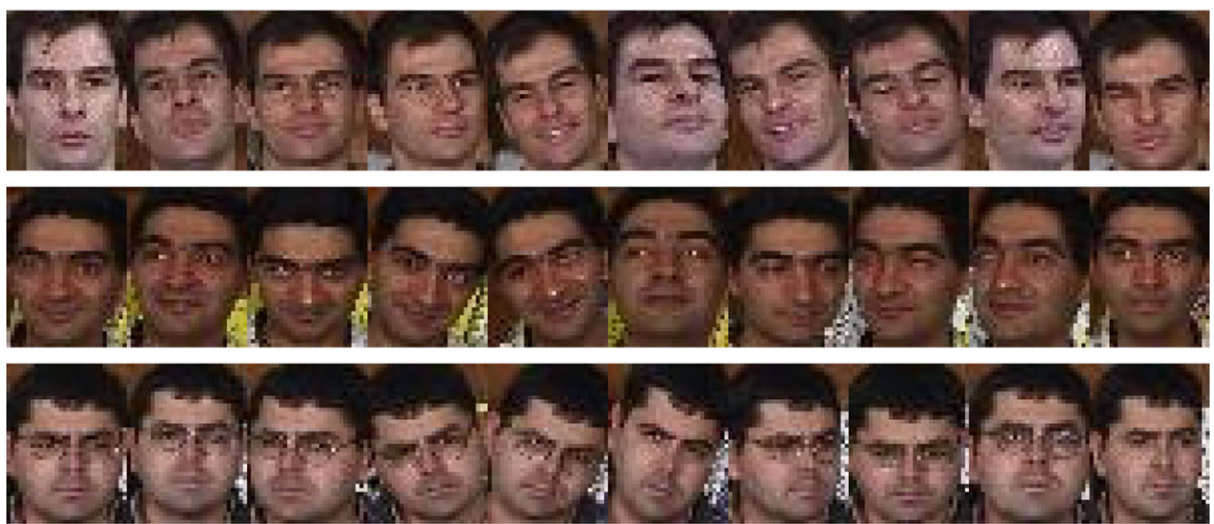

Fig. 12 Some training images and test images of Georgia Tech face database

\subsection{Experiments on FERET face database}

Next, the FERET face database [39] is used to conduct the experiment. This subset has 200 classes and each class has 7 images. We took the first 1, 2, 3, 4 image of each class as the training samples and the remainder as the test sample. Moreover, we set the parameter $K$ to 100. Figure 13 shows the first three face image on FERET face database. In Table 4,we conclude that when the number of training sample is 3 , INNC obtains the highest recognition rate. However, our method performs better than the other eleven methods if the number of the training samples is 1,2 and 4 .

\subsection{Experiments on the CMU-PIE face database}

Then, the subset of CMU-PIE face database [43] which has 3332 images is used to conduct the experiment. Each face has 68 images. We took the first 1, 2, 3, 4 images of each face as the training images and the rest as the test images. Moreover, the parameter $K$ of our method was set to 20. Figure 14 shows some images of CMU-PIE face database and Table 5 shows the experimental results. In Table 5, we conclude that when the number of the training samples is 1 , the recognition rate of DSRM is the highest. However, the proposed method performs better than those eleven state-of-the-art methods when the number of the training samples is 2,3 and 4 .

Table 3 The accuracies (\%) on Georgia Tech face database

\begin{tabular}{lllll}
\hline No. of the training sample & 1 & 2 & 3 & 4 \\
\hline SRC & 37.86 & 46.46 & 50.50 & 57.27 \\
LRC & 34.00 & 47.80 & 53.83 & 57.47 \\
CRC & 37.14 & 45.45 & 50.05 & 53.27 \\
INNC & 33.14 & 39.85 & 40.83 & 43.82 \\
CFKNNC & 38.71 & 47.69 & 53.67 & 54.73 \\
Homotopy & 34.00 & 47.80 & 53.83 & 57.47 \\
PLAM & 38.34 & 49.54 & 55.33 & 59.91 \\
The method [54] & 32.29 & 40.92 & 41.83 & 43.27 \\
DSRM & 37.14 & 46.15 & 49.00 & 55.09 \\
BDLRR & 35.43 & 48.46 & 55.33 & 60.09 \\
The method [27] & 33.41 & 47.54 & 48.50 & 53.45 \\
Our method & $\mathbf{3 9 . 0 0}$ & $\mathbf{5 0 . 7 7}$ & $\mathbf{5 6 . 6 7}$ & $\mathbf{6 0 . 5 5}$ \\
\hline
\end{tabular}



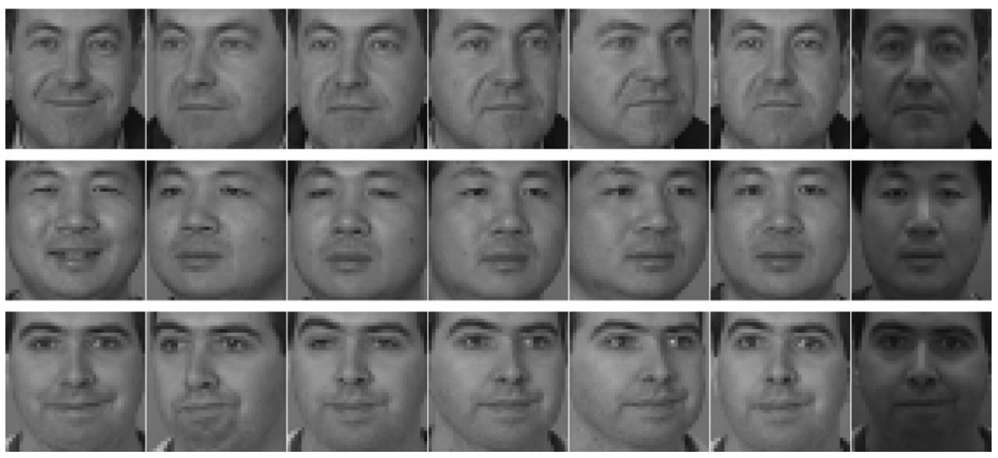

Fig. 13 The first three faces on the FERET face database

\subsection{Experiments on the Libor face database}

Finally, we use Libor face database (http://cswww.essex.ac.uk/mv/allfaces/index.html) to conduct the experiments. Libor face database was designed and maintained by Dr. Libor Spaeck in 2017. The face database has 3040 face images and each face has 20 images. The first 1, 2, 3 and 4 images of each face were taken as training samples and the remainder samples of the face as test samples. Moreover, we set the parameter $K$ to 50 . Some training samples and test samples of Lirbor face database were showed in Fig. 15. From Table 6, we conclude that when the number of the training samples is 4 , the recognition accuracy of DSRM is the highest. However, our method performs better than those eleven state-of-the-art methods when the number of the training samples is 1,2 and 3.

\subsection{Discussion and analysis}

Above mentioned competing methods can be categorized into five groups. SRC, LRC and CRC are traditional sparse representation-based classification method. INNC and CFKNNC are based on nearest neighbor classification method (NNC) [12]. Different from traditional sparse representation-based classification method, Homotopy and PLAM exploit greedy strategy approximation to solve sparse representation problem. So, Homotopy and PLAM are regarded as iterative classification method. The method in [27, 54] belong to the grouped

Table 4 The Accuracies (\%) on the feret face database

\begin{tabular}{lllll}
\hline No. of the training sample & 1 & 2 & 3 & 4 \\
\hline SRC & 50.25 & 64.80 & 60.00 & 53.50 \\
LRC & 44.92 & 64.20 & 59.62 & 76.50 \\
CRC & 44.33 & 58.40 & 44.37 & 55.33 \\
INNC & 44.33 & 58.30 & 50.50 & 54.00 \\
CFKNNC & 47.83 & 63.30 & 54.88 & 57.32 \\
Homotopy & 25.75 & 33.75 & 35.36 & 41.67 \\
PLAM & 22.17 & 30.90 & 37.58 & 45.85 \\
The method [54] & 31.25 & 61.60 & 55.13 & 58.17 \\
DSRM & 36.38 & 52.60 & 48.38 & 61.33 \\
BDLRR & 25.58 & 49.80 & 62.00 & 72.50 \\
The method [27] & 40.08 & 54.30 & 52.88 & 56.00 \\
Our method & $\mathbf{5 1 . 2 5}$ & $\mathbf{6 7 . 2 0}$ & $\mathbf{6 0 . 7 5}$ & $\mathbf{7 7 . 3 7}$ \\
\hline
\end{tabular}



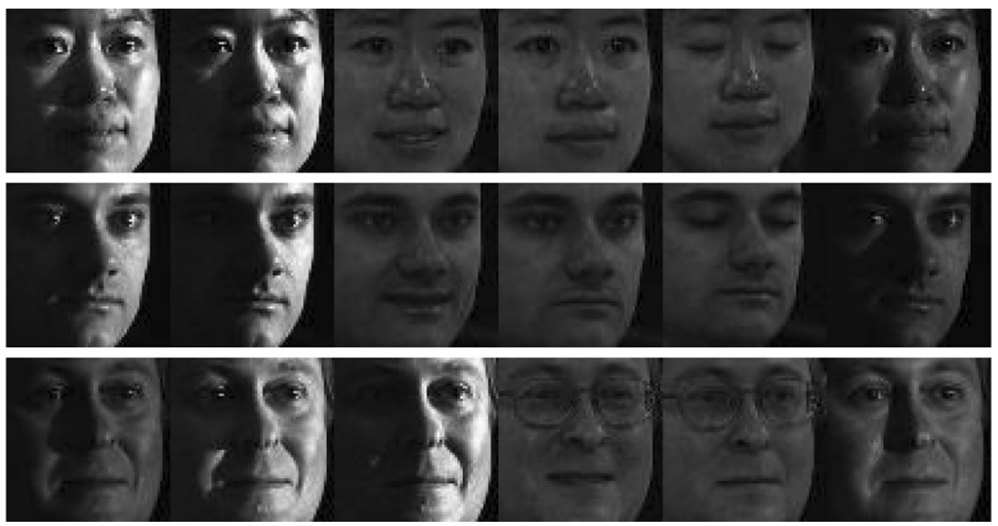

Fig. 14 Some images of CMU-PIE face database

sparse representation-based classification method. And DSRM and BDLRR are the most advanced related method published in recent years.

Through the observation of above experiment results, in most cases, our method is able to obtain higher recognition accuracy than the other competing methods. In fact, our method can be viewed as an improved SRC. Compared with the mentioned three traditional spare representation-based classification methods and two improved NNCs, the improvement in recognition accuracy of our method is obvious. The maximum improvement is greater than 23\%. Although Homotopy and PLAM may be able to obtain higher recognition accuracies than our method if the number of iteration is increased, the time complexity will be increased exponentially. The same as our method, the methods in [27, 54] both exploit the original training set to generate a kind of virtual samples. Then a grouped sparse strategy and weighted fusion approach are used to classify the test sample. Moreover, in the step of fusing the residuals of a class, the method [27] uses the inner product of residual vectors of a class to reflect the relations of different groups. However, the two methods are beat by our method. This reflects that our analysis in the fourth section is rational. For DSRM and BDLRR, when the number of training samples is small, i.e., less than 4 , in most cases, the recognition accuracies of our method are higher than these two most advanced related methods. However, DSRM and BDLRR can beat our method when each class has large number training samples.

Table 5 The accuracies (\%) on the cmu-pie face database

\begin{tabular}{lllll}
\hline No. of the training sample & 1 & 2 & 3 & 4 \\
\hline SRC & 17.92 & 50.22 & 52.14 & 79.51 \\
LRC & 16.85 & 48.94 & 52.46 & 66.70 \\
CRC & 18.17 & 50.47 & 53.07 & 80.03 \\
INNC & 18.17 & 46.45 & 49.11 & 79.38 \\
CFKNNC & 19.38 & 51.07 & 53.00 & 80.92 \\
Homotopy & 34.24 & 49.58 & 51.70 & 79.93 \\
PLAM & 23.16 & 50.13 & 53.23 & 77.24 \\
& 20.62 & 51.77 & 55.06 & 82.52 \\
DSRM The method [54] & $\mathbf{3 5 . 3 5}$ & 51.25 & 53.30 & 81.36 \\
BDLRR & 30.27 & 46.25 & 50.58 & 82.17 \\
& 13.69 & 50.01 & 61.32 & 73.63 \\
Our method & 20.53 & $\mathbf{5 2 . 0 3}$ & $\mathbf{6 3 . 4 2}$ & $\mathbf{8 3 . 3 5}$ \\
\hline
\end{tabular}



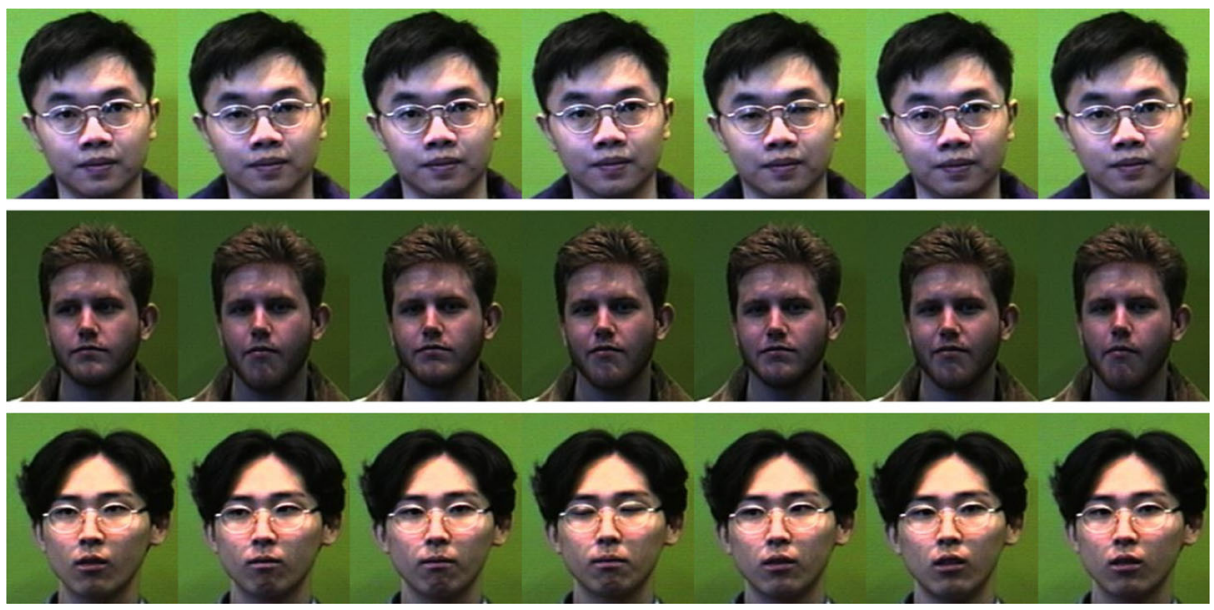

Fig. 15 Some training samples and test samples of Libor face database

In recent years, deep learning theory $[36,45]$ has become a hot spot of research in face recognition. However, deep learning theory need a large number of training samples to train the model. If the number of training sample is very limited, deep learning theory may be over fitting. In this case, deep learning theory is not suitable for the database with a small number of samples. Different from deep learning, our method has a good performance on the small size database.

Our method uses the pixels of face image as the features to classify the test sample. However, the time complexity of our method will be increased accordingly if the size of face image is large. Moreover, a framework of our method exploits the axis-symmetrical property of face to generate a kind of virtual samples. In this case, if the face image is not-symmetrical, the framework may generate a misshapen face image. Fortunately, pervious literatures $[50,51]$ show that image feature extraction can greatly reduce the dimensionality of data without reducing the recognition rate. So our method maybe obtains good recognition accuracy and lower time complexity if it combines with efficient feature extraction methods.

Meanwhile, pervious literatures $[1,2,25]$ show that image feature can be used to steganography $[24,28]$ and digital-watermarking [9]. In this case, maybe the integration of our method and appointed feature extraction method can be used in security certification $[21,22]$ or information hiding $[5,6]$.

Table 6 The accuracies (\%) on the libor face database

\begin{tabular}{lllll}
\hline No. of the training sample & 1 & 2 & 3 & 4 \\
\hline SRC & 86.70 & 87.39 & 87.54 & 87.58 \\
LRC & 89.75 & 91.25 & 91.45 & 91.82 \\
CRC & 86.98 & 87.39 & 87.73 & 88.08 \\
INNC & 87.50 & 88.85 & 89.67 & 90.25 \\
CFKNNC & 82.15 & 82.33 & 84.75 & 86.89 \\
Homotopy & 88.12 & 89.27 & 91.07 & 88.50 \\
PLAM & 90.58 & 90.53 & 91.20 & 89.75 \\
The method [54] & 87.22 & 88.30 & 88.20 & 88.57 \\
DSRM & 91.74 & 92.25 & 93.08 & $\mathbf{9 6 . 6 8}$ \\
BDLRR & 91.94 & 92.23 & 92.78 & 96.08 \\
The method [27] & 86.88 & 88.12 & 88.00 & 88.28 \\
Our method & $\mathbf{9 2 . 1 7}$ & $\mathbf{9 2 . 5 2}$ & $\mathbf{9 3 . 9 9}$ & 94.17 \\
\hline
\end{tabular}




\section{Conclusion}

In this paper, we proposed a novel group representation-based classification method. The proposed method first combines all training samples of the nearest neighbor classes of test sample to form the first group. Next, a framework is performed on the first group to generate a kind of virtual samples. The virtual samples are combined to form the second group. Then, the proposed method performs SRC on these two groups to obtain two residuals for the classes. Finally, a novel weighted fusion approach is used to fuse two residuals and the distance of a class to recognize the test samples. Two residuals include the discriminant information of the test sample in the first and second group, respectively. The distance simultaneously includes the discriminant information of the test sample in two groups. In the subsection of 4.2.1, we can see that the virtual samples of our method are able to reflect the possible change of poses, facial expressions and illuminations of face image well. Moreover, according to the figures of the subsection of 4.2.2 and 4.2.3, we conclude that our method has strong representation ability on classifying the test sample. Experimental results on ORL, Georgia-Tech, FERET, CM-PIE and Libor face databases reflects above conclusion is reasonable.

Acknowledgments This work is supported by the National Natural Science Foundation of China (No.61672333, 61402274, 61461025), China Postdoctoral Science Foundation Special project (No.2014 T70937), the Program of Key Science and Technology Innovation Team in Shaanxi Province (No.2014KTC-18), the Key Science and Technology Program of Shaanxi Province, China (No. 2016GY-081), the Fundamental Research Funds for the Central Universities (No.GK201603083), Interdisciplinary Incubation Project of Learning Science of Shaanxi Normal University, Experimental Technology Research Project of Shaanxi Normal University (No. SYJS201314, SYJS201330).

Open Access This article is distributed under the terms of the Creative Commons Attribution 4.0 International License (http://creativecommons.org/licenses/by/4.0/), which permits unrestricted use, distribution, and reproduction in any medium, provided you give appropriate credit to the original author(s) and the source, provide a link to the Creative Commons license, and indicate if changes were made.

\section{References}

1. Abdul A, Gutub A (2010) Pixel Indicator technique for RGB image steganography. Journal of Emerging Technologies in Web Intelligence (JETWI) 2(1):56-64

2. Abu-Marie W, Gutub A (2010) Hussein Abu-Mansour, image based steganography using truth table based and determinate Array on RGB Indicator. International Journal of Signal and Image Processing (IJSIP) 1(3): 196-204

3. Alassaf N, Alkazemi B, Gutub A (2017) Applicable light-weight cryptography to secure medical data in iot systems. Journal of Research in Engineering and Applied Sciences (JREAS) 2(2):50-58

4. Alharthi N, Gutub A (2017) Data visualization to explore improving decision-making within hajj services. Scientific Modelling and Research 2(1):9-18

5. Al-Otaibi N, Gutub A (2014) Flexible Stego-System for Hiding Text in Images of Personal Computers Based on User Security Priority, Proceedings of 2014 International Conference on Advanced Engineering Technologies (AET-2014), pp. 250-256

6. Al-Otaibi N, Gutub A (2014) 2-Leyer security system for hiding sensitive text data on personal computers. Lecture Notes on Information Theory 2(2):151-157

7. Asif MS, Romberg J (2012) Fast and accurate algorithms for re-weighted $l_{1}$-norm minimization. IEEE Trans Signal Process 61(23):5905-5916

8. Baum M On two types of deviation from the matching law: bias and undermatching. Experimental Analysis of Behavior. https://doi.org/10.1901/jeab.1974.22-231

9. Chen B, Wornell GW (2001) Quantization index modulation: a class of provably good methods for digital watermarking and information embedding. IEEE Information Theory Society 47(4):1423-1443 
10. Chen W, Er M, Wu S (2016) Illumination compensation and normalization for robust face recognition using discrete cosine transform in logarithm domain. IEEE Trans Syst Man Cybern B, Cybern 36(2):458-466

11. Chen B, Yang Z, Huang S, Du X, Cui Z, Bhimani J, Xie X, Mi N (2017) Cyber-Physical System Enabled Nearby Traffic Flow Modelling for Autonomous Vehicles

12. Cover TM, Hart PE (1967) Nearest neighbor pattern classification. IEEE Trans Inform Theory 13(1):21-27

13. Ding M, Fan G (2015) Multilayer Joint Gait-Pose Manifolds for Human Gait Motion Modeling. IEEE Transactions on Cybernetics 45(11):2413-2424

14. Ding M, Fan G (2016) Articulated and generalized Gaussian kernel correlation for human pose estimation, in. IEEE Trans Image Process 25(2):776-789

15. Du B, Wang Z, Zhang L, Zhang L, Liu W, Shen J, Tao D (2015) Exploring representativeness and Informativeness for active learning. IEEE Transactions on Cybernetics 47(1):14-26

16. Ekman P, Hager JC, Friesen WV (1981) The symmetry of emotional and deliberate facial actions. Psychophysiology 18(2):101-106

17 Georghiades AS, Belhumeur PN, Kriegman D (2001) From Few to Many: Illumination Cone Models for Face Recognition under Variable Lighting and Pose. IEEE Trans. Pattern Anal. Mach. Intell. 23(6):643-660.

18. Georghiades S, Belhumeur N, Kriegman D (2001) From few to lighting and pose. IEEE Trans Pattern Anal Mach Intell 23(6):643-660 many: Illumination cone models for face recognition under variable

19. Goel N, Bebis G, Nefian A (2005) Face recognition experiments with random projection. Proc SPIE 5779: 426-437

20. Gong C, Tao D, Liu W, Liu L, Yang J (2017) Label propagation via teaching-to-learn and learning-to-teach. IEEE Transactions on Neural Networks \& Learning Systems 28(6):1452-1465

21. Gutub A (2015) Exploratory Data Visualization for Smart Systems, Smart Cities 2015 - 3rd Annual Digital Grids and Smart Cities Workshop, Burj Rafal Hotel Kempinski, Riyadh, Saudi Arabia

22. Gutub A (2015) Social Media \& its Impact on e-governance, ME Smart Cities 2015-4th Middle East Smart Cities Summit, Pullman Dubai Deira City Centre Hotel, Dubai, UAE

23. Gutub A, Alharthi N (2016) Improving Hajj and Umrah Services Utilizing Exploratory Data Visualization Techniques, Hajj Forum 2016 - the 16th scientific Hajj research Forum, Organized by the Custodian of the Two Holy Mosques Institute for Hajj Research, Umm Al-Qura University - King Abdulaziz Historical Hall, Makkah, Saudi Arabia, 24-25

24. Gutub A, Ankeer M, Abu-Ghalioun M, Shaheen A, Alvi A (2008) Pixel Indicator high capacity Technique for RGB image Based Steganography, WoSPA 2008 - 5th IEEE International Workshop on Signal Processing and its Applications, University of Sharjah, U.A.E, PP. 18-20

25. Gutub A, Al-Qahtani A, Tabakh A (2009) Triple-A: Secure RGB Image Steganography Based on Randomization, AICCSA-2009 - The 7th ACS/IEEE International Conference on Computer Systems and Applications, pp. 400-403

26. Jain A, Nandakumar K, Ross A (2005) Score normalization in multimodal biometric systems. Pattern Recogn 38(12):2270-2285

27. Ke J, Peng Y, Liu S, Wu J, Qiu G (2017) Sample partition and grouped sparse representation. J Mod Opt 64(21):2289-2297

28. Khan F, Gutub A (2007) Message Concealment Techniques using Image based Steganography, The 4th IEEE GCC Conference and Exhibition, Gulf International Convention Centre, Manamah, Bahrain, pp. 2007

29. Lai Z, Wong W, Xu Y, Yang J, Tang J, Zhang D (2016) Approximate orthogonal sparse embedding for dimensionality reduction. IEEE Transactions on Neural Networks and Learning Systems 27(4):723-735

30. Lai Z, Xu Y, Yang J, Shen L, Zhang D Rotational invariant dimensionality reduction algorithms. IEEE Transactions on Cybernetics. https://doi.org/10.1109/TCYB.2016.2578642

31. Liu T, Tao D (2016) Classification with noisy labels by importance reweighting. IEEE Trans Pattern Anal Mach Intell 38(3):447-461

32. Liu W, Wang Y, Li SZ, Tan T (2004) Nearest intra-class space classifier for face recognition, in. Proceedings of the International Conference on Pattern Recognition (ICPR) 4:495-498

33. Liu S, Peng Y, Ben X, Yang W, Qiu G (2016) A novel label learning algorithm for face recognition. Signal Process 124:141-146

34. Liu S, Zhang X, Peng Y, Cao H (2016) Virtual images inspired consolidate collaborative representation based classification method for face recognition. J Mod Opt 63(12):1181-1188

35. Liu Z, Qiu Y, Peng YP, Zhang J (2017) Quaternion based maximum margin criterion method for color face recognition. Neural Process Lett 45(3):913-923

36. Liu Z, Luo P, Wang X, Tang X. Deep Learning Face Attributes in the Wild, ICCV, pp. 1-9

37. Naseem I, Togneri R, Bennamoun M (2010) Linear regression for face recognition. IEEE Trans Pattern Anal Mach Intell 32(11):2106-2112 
38. Niyogi P, Girosi F, Poggio T (1998) Incorporating prior information in machine learning by creating virtual examples. Proc IEEE 86(11):2196-2209

39. Phillips P, Moon H, Rizvi S, Rauss J (2000) The FERET evaluation methodology for face-recognition algorithms. IEEE Trans Pattern Anal Mach Intell 22(10):1090-1104

40. Ryu Y, Oh S (2002) Simple hybrid classifier for face recognition with adaptively generated virtual data. Pattern Recogn Lett 23(7):833-841

41. Samaria F, Harter A (1994) A, parameterization of a stochastic model for human face Identication. In Proceedings of 2nd IEEE Workshop Applications Computer Vision 557(4):138-142

42. Sharma A, Dubey A, Tripathi P, Kuma V (2010) Pose invariant virtual classifiers from single training image using novel hybrid-eigenfaces. Neurocomputing 73(10-12):1868-1880

43. Sim T, Baker S, Bsat M (2002) The CMU pose, illumination, and expression (PIE) database. In Proceedings of the Fifth IEEE International Conference on Automatic Face and Gesture Recognition 435(19):46-51

44. Song Y, Kim Y, Chang U, Kwon H (2006) Face recognition robust to left/right shadows; facial symmetry. Pattern Recogn 39(8):1542-1545

45. Sun Y, Wang X, Tang X. Deep Learning Face Representation from Predicting 10,000 Classes, CVPR, pp. 1-8

46. Tan X, Chen S, Zhou Z, Zhang F (2006) Face recognition from a single image per person: a survey. Pattern Recogn 39(9):1725-1745

47. Thian N, Marcel S, Bengio S (2003) Improving face authentication using virtual sample, Proceeding of the IEEE International Conference on Acoustics, Speech, and Signal Processing. pp. 6-10

48. Wang Q, Zhang X, Wu Y, Tang L, Zha Z (2017) Non-convex weighted $l_{p}$ minimization based group sparse representation framework for image Denoising. IEEE Signal Processing Letters 99. https://doi.org/10.1109 /LSP.2017.2731791

49. Wen X, Wen J (2016) Improved the minimum squared error algorithm for face recognition by integrating original face images and the mirror images. Optik-International Journal for Light and Electron Optics 127(2):883-889

50. Wen J, Fang X, Cui J (2018) Robust sparse linear discriminant analysis, IEEE Transactions on Circuits and Systems for Video Technology

51. Wen J, Xu Y, Li Z (2018) Z, inter-class sparsity based discriminative least square regression. Neural Netw 102:36-47

52. Wright J, Yang A, Ganesh A, Sastry S, Yi M (2009) Robust face recognition via sparse representation. IEEE Trans Pattern Anal Mach Intell 31:210-227

53. Xu Y, Zhang D, Yang J, Yang J (2011) A two-phase test sample sparse representation method for use with face recognition. IEEE Transactions on Circuits and Systems for Video Technology 21(9): 1255-1262

54. Xu Y, Zhu Z, Li Z, Liu G, Lu Y, Liu H (2013) Using the original and 'symmetrical face' training samples to perform representation based two-step face recognition. Pattern Recogn 46(4):151-1158

55. Xu Y, Zhu Q, Chen Y, Pan J (2013) An improvement to the nearest neighbor classifier and face recognition experiments. International Journal of Innovative Computing, Information and Control 9(2):543-554

56. Xu Y, Zhu Q, Fan Z, Qiu M, Chen Y, Liu H (2013) Coarse to fine K nearest neighbor classifier. Pattern Recogn Lett 34:980-986

57. Xu Y, Li X, Yang J, Zhang D (2014) Integrate the original face image and its mirror image for face recognition. Neurocomputing 131:191-199

58. Xu Y, Zhang B, Zhong Z (2015) Multiple representations and sparse representation for image classification. Pattern Recogn Lett 68:9-14

59. Xu Y, Zhong Z, Yang J, You J, Zhang D, New Discriminative A (2017) Sparse representation method for robust face recognition via L2 regularization. IEEE Transactions on Neural Networks and Learning Systems 28(10):2233-2242

60. Yang AY, Zhou Z, Balasubramanian AG, Sastry SS, Ma Y (2013) Fast $l_{1}$-minimization algorithms for robust face recognition. IEEE Trans Image Process 22(8):3234-3246

61. Yin J, Liu Z, Jin Z, Yang W (2012) Kernel sparse representation based classification. Neurocomputing 77(1):120-128

62. Yu H, Gao L, Li W, Du Q, Zhang B (2017) Locality sensitive discriminant analysis for group sparse representation-based hyperspectral imagery classification. IEEE Geosci Remote Sens Lett 14(8): $1358-1362$

63. Zhang L, Yang M, Feng X (2011) Sparse representation or collaborative representation: Which helps face recognition, International Conference on Computer Vision, pp. 471-478

64. Zhang Z, Xu Y, Yang J, Li X, Zhang D (2015) A survey of sparse representation: algorithms and applications. IEEE Access 3:490-530 
65. Zhang X, Peng Y, Liu S, Wu J, Ren P (2017) A supervised dimensionality reduction method based sparse representation for face recognition. J Mod Opt 64(8):799-806

66. Zhang Z, Xu Y, Shao L, Yang J (2017) Discriminative block-diagonal representational learning for image recognition. IEEE Transactions on Neural Networks and Learning systems. https://doi.org/10.1109 /TNNLS.2017.2712801

67. Zhang G, Zou W, Zhang X, Hu X, Zhao Y (2017) Diversity and adaptive weighted fusion for face recognition. Digital Signal Processing 62:150-156

68. Zhang B, Xu Y, Yang J (2018) Adaptive weighted nonnegative low-rank representation. Pattern Recogn 81: $326-340$

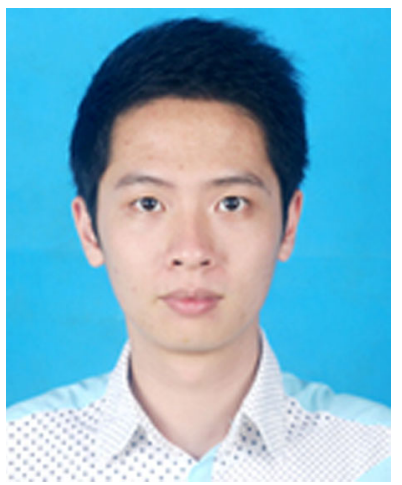

JingCheng Ke received the B.S. degree in Computer Science and Technology from Southwest Minzu University, Chengdu, China, in 2014, and the M.S. degree in computer science from Shaanxi Normal University, Xian, China, in 2018. His current research interests include pattern recognition and image processing.

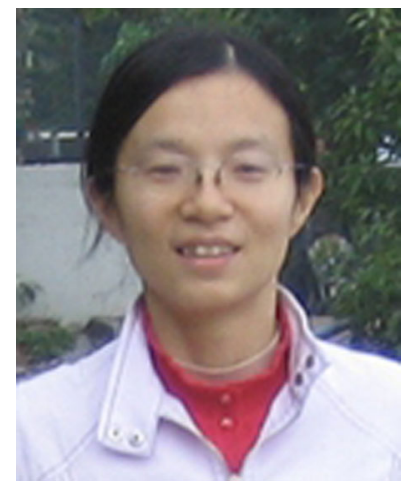

Yali Peng received the M.S. degree in Harbin Engineering University, Harbin, China, in 2005, and the Ph.D. degree in Xi'an Electronic and Science University, Xi'an, China, in 2013. Her current research interests include pattern recognition and machine learining. 